\title{
(2) OPEN ACCESS \\ Proactive response and innovative approaches to SARS-CoV-2 in Vietnam
}

\author{
Nguyen Van Vinh Chau, ${ }^{1}$ Nguyen Tri Dung, ${ }^{2}$ Ronald Geskus, ${ }^{3,4}$ \\ Nguyen Thanh Dung, ${ }^{1}$ Le Manh Hung, ${ }^{1}$ Pham Quang Thai, ${ }^{5,6}$ \\ Tran Tan Thanh, ${ }^{3}$ Nguyen Thanh Truong, ${ }^{1}$ Lam Minh Yen, ${ }^{3}$ \\ Guy Thwaites, ${ }^{3,4}$ Dang Duc Anh, ${ }^{5}$ Le Van Tan (1) ${ }^{3}$
}

${ }^{1}$ Hospital for Tropical Diseases, Ho Chi Minh City, Vietnam ${ }^{2}$ Center for Disease Control and Prevention, Ho Chi Minh City, Vietnam

${ }^{3}$ Oxford University Clinical Research Unit, Ho Chi Minh City, Vietnam

${ }^{4}$ Centre for Tropical Medicine and Global Health, Nuffield Department of Medicine, University of Oxford, Oxford, UK ${ }^{5}$ National Institute of Hygiene and Epidemiology, Ha Noi, Vietnam

${ }^{6}$ Hanoi Medical University, School of Preventive Medicine and Public health, $\mathrm{Ha}$ Noi, Vietnam

Correspondence to Dr Le Van Tan, Oxford University Clinical Research Unit, Ho Chi Minh City 700000, Viet Nam: tanlv@oucru.org

Received 1 March 2021 Accepted 21 March 2021 Published Online First

\section{COUNTRY OVERVIEW AND TIMELINES OF COVID-19 OUTBREAK IN VIETNAM}

Vietnam is a lower-income to middleincome country with a population of 97 million. In 2018, its gross domestic product per capita was US\$2.566, and around US $\$ 150$ per person were spent on healthcare. The healthcare system in Vietnam includes both public and private providers, with less than one doctor and two nurses per 1000 population. Public hospitals are divided into four levels: central, provincial, district and commune levels, and are responsible for providing healthcare for the majority of the residents in Vietnam.

The country has a $1300 \mathrm{~km}$ border with China in the north, where SARS-CoV-2 first emerged. ${ }^{1}$ Therefore, Vietnam was at high risk of SARS-CoV-2 infection as the virus spread outside of China in early 2020. Yet, as of 23 February 2021, there have been only a total of 2401 reverse transcription (RT)-PCR-confirmed cases of SARS-CoV-2 infection with three major waves attributable to locally acquired infection (figure 1) and 35 deaths from the virus reported in Vietnam. ${ }^{2}$ The outbreak in the coastal city of $\mathrm{Da}$ Nang between July and August 2020 represented a major outbreak associated with community transmission. ${ }^{34}$ This was then followed by the detection of sporadic cases of community transmission in the last quarter of 2020. In the morning of 28 January 2021 , after 55 days of no community transmission anywhere in the country, two cases of locally acquired infection were reported in two neighbouring provinces (Hai

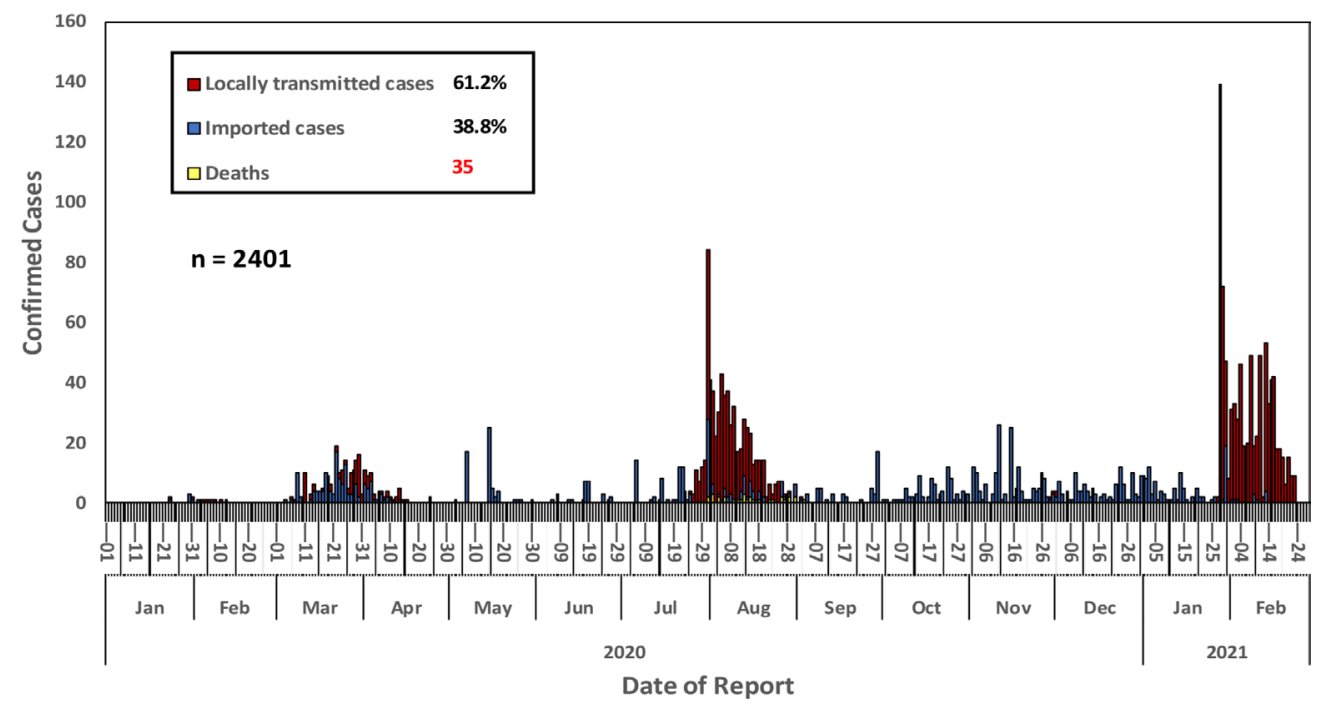

Figure 1 Outbreaks of COVID-19 in Vietnam with three waves of community transmission (source: who.int/vietnam). 
Duong and Quang Ninh). By the end of the same day, the number of infections climbed to 88 , marking the beginning of the third wave of community transmission in Vietnam. Although as of 23 February 2021, a total 809 infections have been reported from the current outbreak, the epidemic curve is now falling (figure 1). Likewise, the number of provinces with ongoing community transmissions has decreased from 13 to 3 since 17 February $2021 .^{25}$

Despite the ongoing challenges, Vietnam is one of the few countries worldwide that have successfully controlled SARS-CoV-2. Here, we discuss the measures taken that have led to the control of SARS-CoV-2 in Vietnam.

\section{RAPID AND COORDINATED PUBLIC HEALTH RESPONSE}

Before the SARS-CoV-2 pandemic, Vietnam had experienced outbreaks of several emerging infectious diseases. They have included the severe acute respiratory syndrome (SARS) caused by SARS-CoV-1 in 2003, bird influenza caused by influenza A virus subtype H5N1 in 2004 and the $2009 \mathrm{H} 1 \mathrm{~N} 1$ pandemic. Notably, back in 2003, Vietnam was recognised by the WHO as the first country to successfully contain SARS-CoV-1. Additionally, endemic infections such as dengue and hand, foot and mouth disease have continuously posed significant challenges on the healthcare system in Vietnam on a yearly basis. Lessons learnt from these outbreaks and endemic infections were thus critical in informing Vietnam's rapid and coordinated response to SARS-CoV-2. ${ }^{6}$

On 16 January 2020, the Vietnam Ministry of Health $(\mathrm{MOH})$ issued the first national guidelines on COVID-19 diagnostics and management. The guidelines also covered guidance on contact tracing and 14-day isolation of individuals in contact with a PCRconfirmed case. Furthermore, on 20 January 2020, the
$\mathrm{MOH}$ issued the national strategic plan for COVID-19 response with the overarching aims of early detection, rapid containment and effective case management. On 30 January 2020, the government assembled the National Steering Committee for COVID-19. The Committee is responsible for the highest level of COVID-19 response and coordination in Vietnam. On the same day, the $\mathrm{MOH}$ established the National Rapid Response Team, with members drawn from referral hospitals across the country. The team is responsible for providing expert support to primary COVID-19 centres and hospitals across Vietnam.

\section{CENTRALISED PATIENT MANAGEMENT}

Unlike strategies applied in other countries, where only confirmed cases with severe illness are admitted to health facilities for clinical management, any PCRconfirmed cases in Vietnam have been admitted to one of the designated COVID-19 treatment centres/ hospitals deployed across the country since early 2020, regardless of clinical symptoms. Severe cases are subjected to panel discussion via teleconference with participants from the national COVID-19 response team, thus enabling the best clinical management.

\section{EARLY SCHOOL CLOSURES AND ROBUST BORDER CONTROLS}

School closure was initially scheduled for the Lunar New Year holiday between the end of January and early February 2020. However, in response to the introduction of SARS-CoV-2 into Vietnam on 23 January $2020^{7}$ and the escalation of community transmission during of the first wave, school and university closures were extended until early May 2020 when the nationwide outbreak was brought under control.

Vietnam also took early actions to enhance border control measures from as early as January 2020, when the country first heard about the emergence of a cluster

\begin{tabular}{|c|c|}
\hline 3 January 2020 & Strengthening of border control measures \\
\hline 23 January 2020 & Strengthening of border control measures, especially at the border with China; isolation of suspected cases \\
\hline 23 January 2020 & Vietnam cancelled all flights to Wuhan. \\
\hline 25 January 2020 & Health declaration was made mandatory for passengers arriving from China. \\
\hline 29 January 2020 & Suspending flights to and from affected areas in China \\
\hline 1 February 2020 & Suspending flights to and from China, Taiwan, Hong Kong and Macau \\
\hline 24 February 2020 & Health declaration was made mandatory for passengers arriving from Korea \\
\hline 28 February 2020 & Suspending visa-free policy for Korean citizens \\
\hline 1 March 2020 & $\begin{array}{l}\text { Health declaration was made mandatory for passengers arriving from China, Korea, Italy and Iran. Suspected cases with fever, } \\
\text { shortness of breath or cough were subjected to isolation. }\end{array}$ \\
\hline 6 March 2020 & Health declaration was made mandatory for all passengers arriving in Vietnam. \\
\hline 22 March 2020 & 14-day quarantine at centralised facilities was made mandatory for all arriving travellers. \\
\hline 21-23 March 2020 & Suspending flights to and from Singapore, Thailand, Indonesia, Laos, Myanmar, UK and Japan \\
\hline 27 March 2020 & Health-status update was made mandatory to all individuals entering Vietnam from 8 March onward to aid surveillance. \\
\hline 1 April 2020 & Closing of main and auxiliary border gates \\
\hline August 2020 & Repatriation flights arranged for Vietnamese people stuck abroad and entry allowed for highly skilled workers \\
\hline
\end{tabular}


of pneumonia of unknown origin in Wuhan, China (table 1). Additionally, body temperature screening at airports and border gates was applied for passengers arriving in Vietnam from Wuhan where the epidemic first started. This was then extended to arrivals from the whole China and other countries where community transmission was ongoing.

An international travel ban was first applied to Wuhan, China, on 23 January 2020. As the pandemic escalated in the region and beyond, Vietnam closed its borders and banned international flights to and from China, followed by other countries (including Taiwan, Korea, Italy, the UK and the USA). Finally, Vietnam closed all main and auxiliary border gates on 1 April 2020 (table 1).

\section{MASK POLICIES AND 5K MESSAGE}

Wearing a face mask in public was recommended by the government in January 2020 and was then made mandatory on 16 March 2020. On 31 August 2020, the $\mathrm{MOH}$ launched a $5 \mathrm{~K}$ (in Vietnamese) message to help people get used to a new normal state under the COVID-19 pandemic while protecting themselves from the infection. $5 \mathrm{~K}$ stands for 'Khẩu trang' (face masks), 'Khử khuẩn' (disinfection), 'Khoảng cách' (distance), 'Không tụ tập' (no gathering) and 'Khai báo y tế' (health declaration). By launching the $5 \mathrm{~K}$ campaign, the government encouraged people to (1) wear masks in public, quarantine centres and medical facilities; (2) regularly wash hands with soap or sanitisers plus surface cleaning by disinfectants; (3) keep distance from each other, (4) avoid mass gathering and (5) make health declaration.

\section{THIRD-DEGREE CONTACT TRACING}

An innovative and unique strategy to SARS-CoV-2 control in Vietnam has been a meticulous contact-tracing approach, which is referred as third-degree contact tracing (figure 2). According to this approach, a confirmed case is defined as F0, while close contacts of F0 are called F1, and F2s are those in close contact with an F1. Once an F1 is tested positive, F2s become F1 and therefore contacts of F2s (F3s) become F2, and so on.

When a confirmed case (F0) is identified, local health officers conduct an epidemiological investigation by asking the F0 to recall his/her recent close contacts (F1) and travel history since his/her last contact with a confirmed case (if known) or over the last 14 days. Detailed travel history and contacts of F0 are then made publically available on the government COVID-19 website (ncov.moh. gov.vn), local magazines and national TV channels so that people can identify whether they have an epidemiological link with a confirmed case. Supporting this meticulous contact tracing approach is the use of locally developed mobile apps, such as Bluezone (rolled out on 18 April 2020). Bluezone is a Bluetooth-based app, which helps users identify whether they were in close contact with a confirmed case. Individuals with an epidemiological link with F0 are thus encouraged to present to or contact the nearest local hospital for advice on isolation and testing. Once F1s are identified, the same approach is used to determine their contacts or F2, and so on.

\section{MASS QUARANTINE AND TARGETED LOCKDOWN}

In addition to the administration of F0 to COVID-19 treating hospitals for clinical follow-up, F1s are also subjected to a minimum of 14-day isolation at one of the

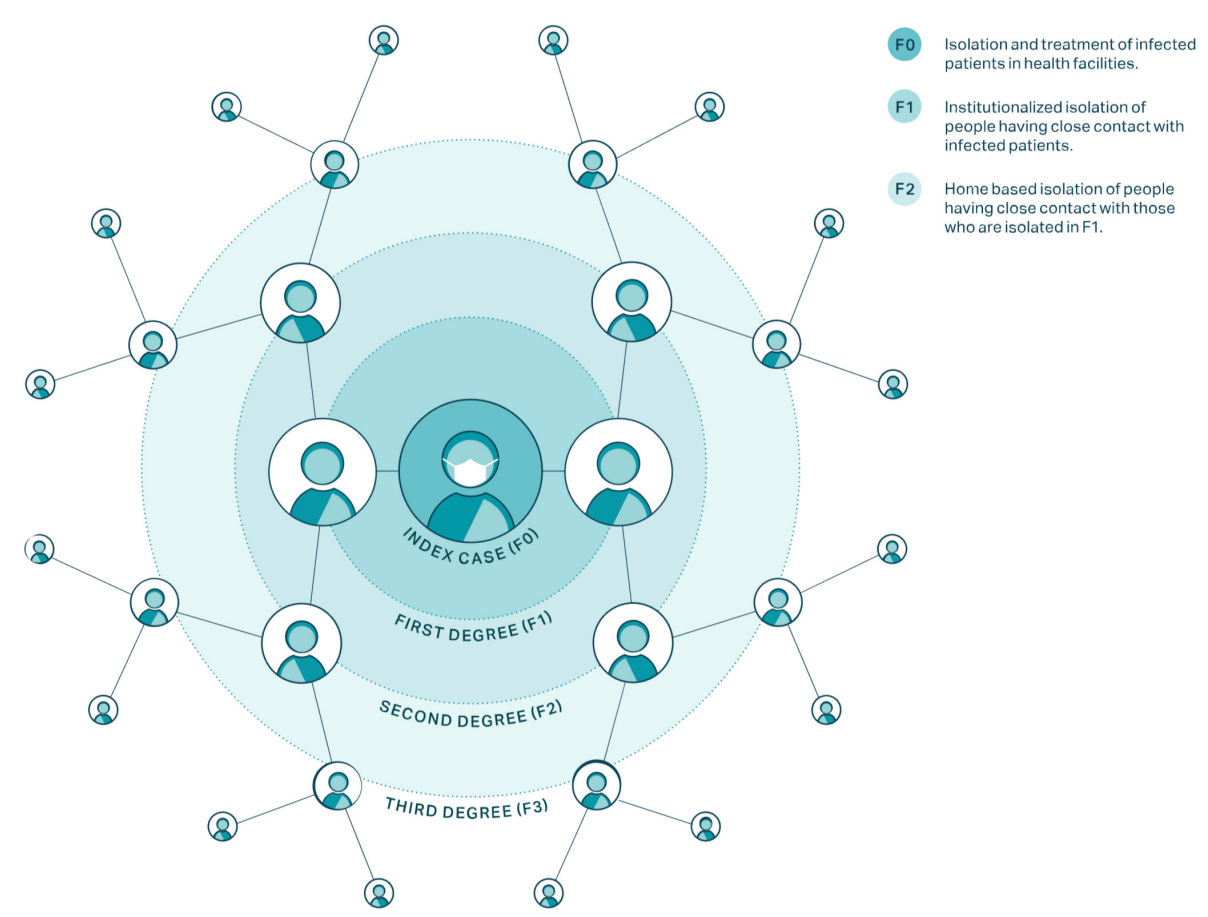

Figure 2 Illustration showing the third-degree contact tracing applied as part of COVID-19 control measures in Vietnam since the beginning of the pandemic (adapted from Pollack et $a l^{4}$ with permission). 
state-run quarantine facilities deployed across Vietnam since early March. Meanwhile, F2s are requested to self-isolate at home for a maximum of 14 days. The same 14-day quarantine at state-run facilities has also been applied to all travellers arriving in Vietnam since early March 2020. As of 21 February 2021, more than 1.3 million people have been quarantined at one of the designated isolation centres across Vietnam. ${ }^{5}$ As the pandemic progressed and scientific evidence about the transmission potential of asymptomatic infection arose, ${ }^{8}$ Vietnam's extensive contact tracing, mass quarantine and wearing a face mask in public proved to be innovative and critical to suppress the community transmission of SARS-CoV-2 in the country.

Alongside the isolation of confirmed cases, their contacts and travellers to Vietnam, Vietnam has also implemented mass quarantine of suspected transmission hotspots (eg, a city, a town or a community). Table 2 lists all major lockdowns implemented by the Vietnamese government as of February 2021. The first community lockdown was applied on 13 February 2020 to Son Loi commune in Vinh Phuc, Northern Vietnam. At that time, there was a cluster of six local infections linked with eight Vietnamese employees returning from Wuhan. ${ }^{9}$ The lockdown was lifted on 3 March, marking the first in-country success of targeted lockdown.

On 1 April 2020, when community transmission was escalating and the total reported cases in Vietnam exceeded 200, the prime minister enacted a 2-week nationwide lockdown. Accordingly, non-essential businesses and public transport were shut down, while only essential travel between cities/provinces was allowed. Gatherings of more than two people in public were prohibited. People were asked not to leave their house unless there was a need for essential activities (eg, buying food or seeking medical care). The lockdown was extended to 30 April for high-risk areas (including Ho Chi Minh city and $\mathrm{Ha}$ Noi).

Most recently, on 28 January 2021, Chi Linh, a city of 220000 households in Hai Duong province in northern Vietnam, was immediately placed under quarantine after the report of 83 new cases of locally acquired infection of unknown origin. Since then, the lockdown has been extended to other towns and villages. On 16 February, Hai Duong province, the epicentre of the ongoing nationwide outbreak, was placed under full lockdown due to the escalation of the in-province outbreak.

\section{INNOVATIVE TESTING STRATEGIES}

One of the key factors determining the success of Vietnam's approach to extensive contact tracing, isolation and targeted lockdown has been the ability of the country to rapidly increase its diagnostic capacity and the innovative way of conducting mass RT-PCR screening. Soon after the diagnosis of the first cases of SARS-CoV-2 in Vietnam in January 2020, the government pushed forward the development of local diagnostic kits for SARS-CoV-2. On 5 March, the first locally produced COVID-19 RT-PCR test kit developed by Military Medical University was

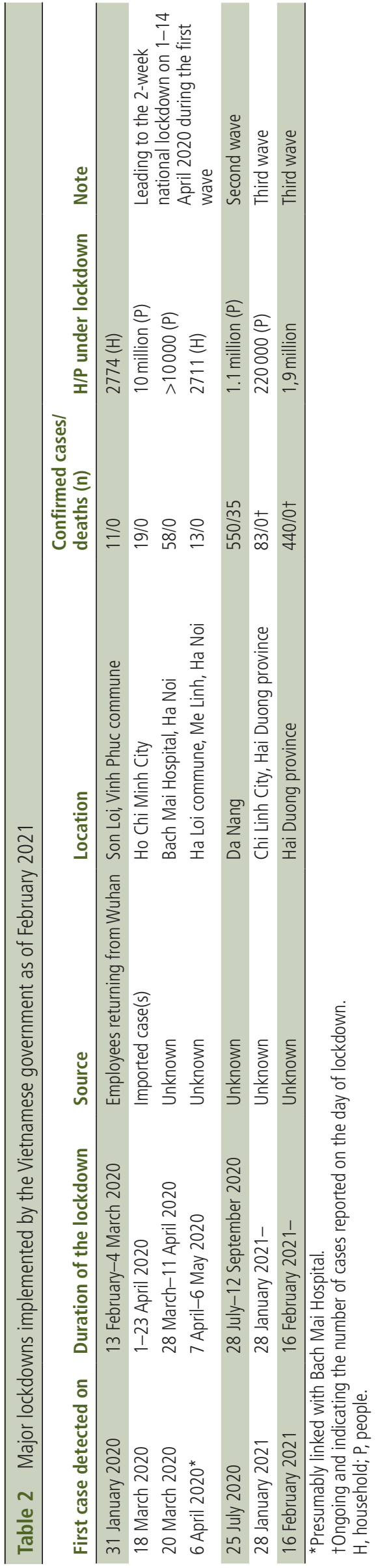




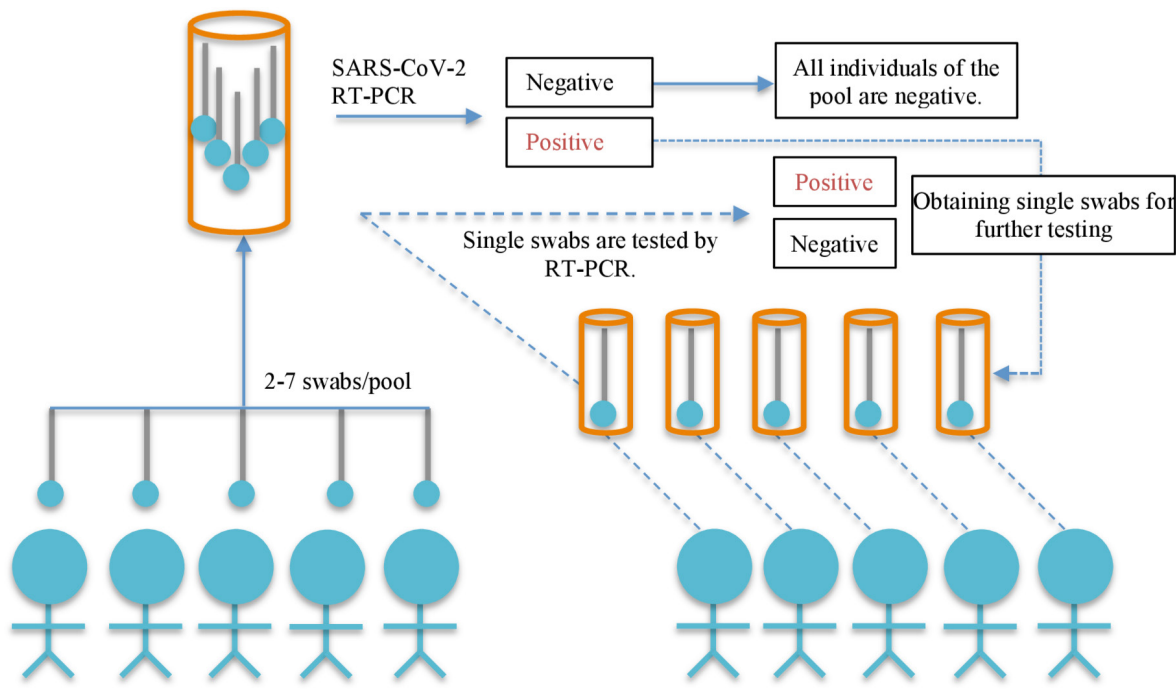

Figure 3 Illustration of the sample pooling strategy that was successfully applied for mass screening of SARS-CoV-2 during the second wave of COVID-19 in Vietnam, July-August 2020. ${ }^{11}$ Nasal-pharyngeal throat swabs from two to seven individuals were combined in a single $15 \mathrm{~mL}$ collection tube containing $3 \mathrm{~mL}$ of viral transport medium for subsequent reverse transcription (RT)-PCR screening for SARS-CoV-2. If SARS-CoV-2 testing of the pool returned positive, single nasal-pharyngeal throat swabs from each individual of the corresponding group were then collected and retested separately for SARS-CoV-2 by RT-PCR.

commercialised by Viet A Corporation. The kit has now been widely used as a screening assay at the majority of COVID-19 diagnostic labs in Vietnam, while WHO recommended RT-PCR protocols serve as confirmatory assays. ${ }^{10}$ Additionally, centres qualified for SARS-CoV-2 RT-PCR testing increased from two sites in early 2020 to 91 across the country by 23 November 2020 . The country's current capacity is around 100000 PCR tests per day. As of 21 February 2021, nearly two million PCR tests have been conducted in Vietnam. ${ }^{5}$

The availability of in-country capacity has allowed Vietnam to integrate mass PCR screening into its national COVID-19 control programme. For example, during the Da Nang outbreak in July-August, mass screening coupled with an innovative sample pooling strategy ${ }^{11}$ was applied. Accordingly, swabs from two to seven individuals living in areas under lockdown were placed in one tube at collection for mass screening (figure 3). In this way, a total of 22 people without any history of contact with a confirmed case were identified after testing nearly 100000 individuals by RT-PCR between 8 and 21 August 2020.This approach contributed to the success of COVID-19 control during the second wave. Most recently, this novel sample pooling strategy has been deployed for mass screening of SARS-CoV-2 as part of the national response to the ongoing outbreak. ${ }^{5}$

\section{INNOVATIVE COMMUNICATIONS}

Keys to the success of SARS-CoV-2 control in Vietnam have been the transparent communications of the Vietnamese government and the compliance of the population with control measures. Since the beginning of the pandemic, the government has used a wide range of innovative communication strategies to keep the public informed with information about the disease, the transmission mechanisms and the in-country preventive responses. Social media, national television, local magazines, national hotline (19009095), mobile short message service (SMS) texts and the official COVID-19 website of the $\mathrm{MOH}$ (ncov.moh.gov.vn) were all used as communication tools.

Since the beginning of the pandemic, $\mathrm{MOH}$ sent regular updates on COVID-19 preventive responses and the national situation via SMS texts and messaging apps such as Zalo. On 9 January, the first COVID-19 news was posted on the government COVID-19 website (ncov. moh.gov.vn). Since then, this website has served as the most reliable and up-to-date source of information on the COVID-19 situation in Vietnam.

In February, a popular Vietnamese song was given new lyrics, 'Ghen Co Vy' (Jealous Coronavirus) to raise public awareness of the disease and to promote good hygiene habits against COVID-19. ${ }^{12}$ In collaboration with the Central Committee of the Vietnam Fatherland Front, the Vietnam Digital Media Association and the United Nations Children's Fund, the $\mathrm{MOH}$ launched the \#Onhavanvui (\#StayHomeIsFun) campaign on TikTok. ${ }^{13}$ In addition to using this approach as a communication channel about COVID-19, the government also aimed to create a virtual playground for the community to stay connected while under the lockdown. Accordingly, TikTok users are encouraged to share their daily activities at home such as cooking (\# onhanoitro), exercising (\#onhakhoemanh), studying (\#onhahocbai) with the community. Remarkably, as of January 2021, the hashtag \#Onhavanvui of the $\mathrm{MOH}$ on TikTok has attracted nearly 11 billion views, while Ghen Co Vy has received some 70 million views. 


\section{CREATIVE APPROACHES INITIATED BY THE COMMUNITY TO SUPPORT VULNERABLE PEOPLE}

Caring for others, especially during a difficult time, has always been part of the Vietnamese culture. As such, during the COVID-19 crisis, Vietnamese people came up with various innovative initiatives to support each other. For example, during the national lockdown in April, entrepreneur Hoang Tuan Anh invented a rice automated teller machine (ATM). The aim was to help vulnerable people with daily meals. These semiautomatic machines dispensed rice for free from a container through a plastic tube with an amount of $1.5 \mathrm{~kg}$ each time. It was first introduced in Ho Chi Minh City and was then expanded to other cities, including Ha Noi and Da Nang. Building on the success of rice ATM, mask and food ATM initiatives were subsequently invented.

\section{CONCLUSIONS}

Early preparedness and proactive coordinated responses encompassing early border closure, physical distancing, face masks, targeted lockdown, contact tracing, isolation and pooled testing coupled with innovative communication and the engagement of the whole society have been keys to the success of SARS-CoV-2 control in Vietnam.

TwitterLe Van Tan @Le_Van_Tan

Acknowledgements The authors thank WHO Vietnam Country Office and Chevy Lazenby from Gates Venture for providing us with high-resolution figures (1 and 2) for reproduction in the present work.

Contributors Conceptual design: NVVC, GT, DDA and LVT; writing the first draft: LVT; review, editing and approval of the manuscript: all authors.

Funding LVT, GT and RG are supported by the Wellcome Trust of Great Britain (204904/Z/16/Z (LVT) and106680/B/14/Z (GT and $R G)$ ).

Competing interests None declared.

Patient consent for publication Not required.

Provenance and peer review Commissioned; externally peer reviewed.

Open access This is an open access article distributed in accordance with the Creative Commons Attribution Non Commercial (CC BYNC 4.0) license, which permits others to distribute, remix, adapt, build upon this work non-commercially, and license their derivative works on different terms, provided the original work is properly cited, appropriate credit is given, any changes made indicated, and the use is non-commercial. See: http://creativecommons.org/ licenses/by-nc/4.0/.

ORCID iD

Le Van Tan http://orcid.org/0000-0002-1791-3901

\section{REFERENCES}

1 Zhu N, Zhang D, Wang W, et al. A novel coronavirus from patients with pneumonia in China, 2019. N Engl J Med 2020;382:727-33.

2 Health, U.i.f.V.M.o. Available: https://ncov.moh.gov.vn/

3 Nguyen TV, Tran QD, Phan LT, et al. In the interest of public safety: rapid response to the COVID-19 epidemic in Vietnam. BMJ Glob Health 2021;6:e04100.

4 Pollack T, Thwaites G, Rabaa M. Emerging covid-19 success story: vietnam's commitment to containment. Examplars in Global Health 2021.

5 Vietnam, W.r.o.f. Viet Nam COVID-19 Situation Report \#30 2021.

6 Van Tan L. COVID-19 control in Vietnam. Nat Immunol 2021;22:261.

7 Phan LT, Nguyen TV, Luong QC, et al. Importation and human-to-human transmission of a novel coronavirus in Vietnam. N Engl J Med 2020;382:872-4.

8 Chau NVV, Thanh Lam V, Thanh Dung N. The natural history and transmission potential of asymptomatic SARS-CoV-2 infection. Clin Infect Dis 2020.

9 Le TQM, Takemura T, Moi ML, et al. Severe Acute Respiratory Syndrome Coronavirus 2 Shedding by Travelers, Vietnam, 2020. Emerg Infect Dis 2020;26:1624-6.

10 Corman VM, Landt O, Kaiser M, et al. Detection of 2019 novel coronavirus (2019-nCoV) by real-time RT-PCR. Euro Surveill 2020;25.

11 Thanh TT, Nhan NTT, Mai HK, et al. The application of sample pooling for mass screening of SARS-CoV-2 in an outbreak of COVID-19 in Vietnam. Am J Trop Med Hyg 2021. doi:10.4269/ ajtmh.20-1583. [Epub ahead of print: 22 Jan 2021].

12 Get the latest information from the MoHFW about COVID-19. Available: https://www.youtube.com/watch?v= BtulL3oArQw

13 TikTok and UNICEF Launch the \#ONhaVanVui Campaign to Encourage Users to stay happy at home infight against COVID-19, 2020. Available: https://www.unicef.org/vietnam/ press-releases/tiktok-and-unicef-launch-onhavanvui-campaignencourage-users-stay-happy-home-infight 